\title{
ESTUDO DE SOBRECARGA POSTURAIS EM ACADÊMICOS DE FISIOTERAPIA DO CENTRO UNIVERSITÁRIO DO PARÁ
}

Tereza Cristina dos Reis FERREIRA ${ }^{1}$

Antonio de Castro TAVARES ${ }^{2}$

Edilson Carlos Sousa LOPES JUNIOR ${ }^{3}$

Fagnor Augusto Maia LOPES ${ }^{4}$

Jamily Planeta Rocha da SILVA ${ }^{5}$

${ }^{1}$ UEPA - Departamento do Movimento Humano-Curso de Fisioterapia. CESUPA -Areas das Ciências Ambientais,Biológicas e da Saúde- Curso de Fisioterapia. tereza reis@ yahoo.com.br

${ }^{2}$ Discente do Centro Universitário do Pará. antonidecastro@ hotmail.com

${ }^{3}$ Discente do Centro Universitário do Pará. edilson_lopes_jr@hotmail.com

${ }^{4}$ Discente do Centro Universitário do Pará. fagnor.augusto@ hotmail.com

${ }^{5}$ Discente do Centro Universitário do Pará. jamily_planeta.rs@ hotmail.com

Recebido em: 22/12/2014 - Aprovado em: 18/06/2015 - Disponibilizado em: 15/07/2015

\begin{abstract}
RESUMO
INTRODUÇAO: A postura pode ser compreendida como a configuração das articulações de um corpo, isto é, o conjunto de ângulos que expressam o arranjo relativo entre os segmentos de um corpo, sendo considerada correta quando é utilizada com máxima eficiência e mínimo esforço. Este trabalho teve como objetivo verificar a incidência de sobrecargas posturais em acadêmicos de Fisioterapia do CESUPA decorrentes da prática de atividade clínica. METODOLOGIA: A captação de imagens foi realizada por meio de uma filmadora para registrar as posturas adotadas durante o atendimento e utilizado o mapa de desconforto corporal para identificar a região de dor e utilizado o método RULA e OWAS para a avaliação das posturas RESULTADOS: foram avaliados 17 acadêmicos, onde 64,7\% sentiam dor em coluna lombar, 35,9\% sentiam dor em coluna torácica, 29,4\% sentiam dor em coluna cervical, 5,8 \% sentiam dor em sacro, 5,8\% sentiam dor em punho no OWAS evidenciou-se 58,5\% das posturas obtendo score final 2 e no RULA 36,6\% encontravam -se no nível 2 e 44\% encontravam-se no nível 3. CONCLUSÃO: Os resultados obtidos neste trabalho nos permitiu observar uma prevalência de dores com prevalências na coluna vertebral e distúrbios posturais nos acadêmicos de Fisioterapia durante suas atividades de trabalho no estágio supervisionado.

Palavras -chave : sobrecargas; posturas ; acadêmico ; fisioterapia; ergonomia

\section{STUDIES IN ACADEMIC OVERLOAD POSTURAL PHYSICAL THERAPY CENTRO UNIVERSITÁRIO DO PARÁ}

\begin{abstract}
Introduction: The posture can be understood as the configuration of the hum Body Joints, This is the Angles Set What express the relative arrangement between the segments of hum Body, Being Considered correct when used with Maximum Efficiency and Minimum effort. This work aimed to verify the incidence of postural overload on CESUPA of Physiotherapy Academic arising from the clinical activity of Practice. METHODOLOGY: The Image capture was performed PER of A camcorder Middle To register as positions taken during the service and Used the body Para discomfort map to identify a region of pain and Used METHOD RULA and OWAS Pará the Evaluation of postures RESULTS : Were evaluated 17 Academic, Where ( $64.7 \%$ ) feel pain in the lumbar column, (35.9\%) had pain in thoracic spine ( $29.4 \%$ ) feel pain in the cervical column, (5.8\%) felt pain in sacrum ( 5.8 ) \% felt pain in FIST not OWAS -If showed $58.5 \%$ of the postures getting final score 2 and not RULA $36.6 \%$ were -If at Level 2 and $44 \%$ found -If at Level 3. CONCLUSION: The results of this study allowed us to observe nos a Prevalence of Sorrows with prevalence of the spine and postural disorders nos Academic Physical Therapy During their work activities not supervised internship .
\end{abstract}

Key- Words : overload ; posture ; academic ; physiotherapy ; ergonomics 


\section{INTRODUÇÃO}

A postura pode ser compreendida como a configuração das articulações de um corpo, isto é, o conjunto de ângulos que expressam o arranjo relativo entre os segmentos de um corpo (DUARTE et al., 2010).

Segundo Maehler (2003), a postura é considerada correta quando é utilizada com máxima eficiência e mínimo esforço, visto que raramente o corpo permanece parado mais do que alguns momentos e, com frequência, ele realiza movimento de grande variedade de extensão e direção.

Segundo Marques (2010), a posição sentada quando mantida por longos períodos leva à prolongada sustentação da flexão lombar, redução da lordose nessa região e sobrecarga estática nos tecidos ostemioarticulares da coluna. Esses fatores estão relacionados ao desenvolvimento da dor lombar, visto que a maior parte do peso do corpo está distribuída em uma área de suporte na tuberosidade isquiática, precisando de um apoio correto para diminuição da pressão intra discal. (ZAPATER, 2004).

Há uma certa discussão em torno das consequências negativas do trabalho sentado. Alguns estudos apontam relação dos distúrbios osteomusculares com a postura sentada, principalmente quando é adotada por longos períodos de tempo e associada com trabalhos sedentários ou vibracionais (FONTES et al., 2013).

Diferentemente da posição sentada, a posição em pé é aquela em que o trabalhador apresenta maior gasto energético e maior sobrecarga nas articulações dos membros inferiores, o que pode levar o trabalhador à fadiga muscular rapidamente. A postura em pé exige uma contração aumentada dos músculos e dispêndio aumentado de energia. A cintura pélvica é inclinada para frente devido à tensão nos músculos anteriores da coxa. Esta inclinação juntamente com a compressão exercida pelo peso do corpo sobre a coluna lombar acentua a lordose neste nível. A postura em pé por longos períodos de tempo não deve ser mantida, pois aumenta o risco de dores e lesões na coluna (FONTES et al., 2013).

A postura mais adequada ao trabalhador é aquela que ele escolhe livremente e que pode ser variada ao longo do tempo. A concepção dos postos de trabalho ou da tarefa deve favorecer a variação de posturas, principalmente a alternância entre a postura sentada e em pé (MORAES, 2013).

Segundo Magalhães (2011) a manutenção de posturas inadequadas pode ocasionar degeneração dos discos intervertebrais da região cervical, hiperlordose cervical e dorsal, dores lombares, cefaléia tensional, fadiga nos olhos, braços e pés, perturbações circulatórias, varizes nas pernas, artrite cervical e nas mãos, 
desigualdade na altura dos ombros e inflamação das bainhas tendinosas, resultando em queda da produtividade de trabalho, custos médicos ou, até mesmo, o abandono da profissão.

Fontes et al. (2013) em seu estudo que teve como objetivo analisar e comparar a sobrecarga mecânica da posição sentada com a posição em pé em 146 funcionários de um empresa, trouxe como resultados que os funcionários que trabalham na posição sentada apresentaram menor sobrecarga postural pelo método RULA em relação aos funcionários que executam suas funções na postura em pé.

Kalsing (2010), traz que dentre os diversos fatores que podem desencadear dores músculos esqueléticas estão os fatores ergonômicos como repetitividade manual, uso de força e vibração nos movimentos e posturas inadequadas. A prática da fisioterapia requer a utilização de muitas tarefas de trabalho intenso no que se refere ao tratamento do paciente, como levantar, abaixar, rotacionar, alongar, executar terapias manuais e manter posições fletidas (GUEDES; MACHADO, 2008).

Machado et al (2009), em seus estudos com o objetivo de verificar a incidência de LER/DORT com fisioterapeutas docentes de uma IES, trouxe como resultado que a região mais acometida foi a coluna lombar, seguido da coluna cervical, punho e mão e ombro e/ou coluna dorsal resultados esses que corroboraram com Guedes e Machado (2008) onde demonstrou em seu estudo que a área em que os acadêmicos de fisioterapia sentiam dores durante $\mathrm{o}$ atendimento eram a região lombar, seguida da região cervical, e em região torácica.

Esse trabalho justifica-se pelo fato da literatura ser escassa acerca do tema, e Guedes e Machado (2008), a partir de sua pesquisa concluíram que apesar dos acadêmicos terem conhecimento dos mecanismos patológicos das lesões e das formas de tratamento, não aplicam tais conhecimentos quando eles mesmos são acometidos, pois continuam trabalhando mesmo na presença de dor ou desconforto, além de existir também uma carga horária a ser cumprida. $\mathrm{O}$ acometimento desses futuros profissionais se dá devido à falta de experiência aliada à vontade de querer se superar, somadas aos ambientes inadequados. O objetivo deste trabalho foi verificar a incidência de sobrecargas posturais em acadêmicos de Fisioterapia do CESUPA decorrentes da prática de atividade clínica.

\section{METODOLOGIA}

Esta pesquisa foi realizada segundo os preceitos da declaração de Helsinque e do código de Nuremberg, respeitadas as Normas de Pesquisa Envolvendo Seres Humanos do Conselho Nacional de Saúde (res. CNS 446/2012). Este trabalho foi uma pesquisa de campo sendo sua natureza descritiva, onde se 
caracteriza por o pesquisador procurar conhecer a realidade e interpretá-la, sem qualquer tipo de interferência para modificala; tendo como interesse descobrir e observar para então poder descrever, classificar e interpretar os fenômenos que vierem acontecer. A pesquisa foi realizada na clínica - Escola de Fisioterapia do Centro Universitário do Pará, no período de agosto a setembro de 2014. Como critérios de inclusão foram acadêmicos do curso de fisioterapia do CESUPA, cursando o $8^{\circ}$ período do curso, em estágio supervisionado na Clínica Escola de Fisioterapia com termo de consentimento livre e esclarecido (TCLE) assinado e critérios de exclusão não ser acadêmico do curso de fisioterapia do CESUPA, não estava cursando o $8^{\circ}$ período do curso, ou se recusou a assinar o TCLE. Para a avaliação foi utilizado à observação visual direta e uma filmadora Sony Cyber-shot DSC-H70 16.1mp para registrar as posturas adotadas pelos acadêmicos durante o atendimento, e para identificar a região de desconforto corporal foi utilizado o mapa corporal e para a avaliação das posturas foram utilizados os métodos RULA e OWAS. O método OWAS é um dos métodos de avaliação postural mais tradicionais sendo então desenvolvido segundo a premissa básica de ser um método simples, porém fidedigno, possibilitando facilidade no seu uso e no seu aprendizado, apresentando os resultados das porcentagens de tempo que o trabalhador permanece em uma postura "boa" e "má", e ainda propiciar o direcionamento para a melhoria do posto de trabalho. O método RULA foi proposto como um método para avaliação rápida dos danos potenciais aos membros superiores, em função da postura adotada. Avaliando a postura do pescoço, tronco e membros superiores (braço, antebraço e mãos) e relacionando com o esforço muscular e a carga externa a que o corpo está submetido (CARDOSO, 2006).

\section{RESULTADOS}

A casuística inicial era de 20 discentes regularmente matriculados, respeitando aspectos éticos os 3 autores se excluíram da amostra, tendo casuística final 17 discentes do último ano do Curso de Fisioterapia, avaliados por meio do mapa de desconforto corporal, RULA e OWAS. A média de idade foi 22,1 anos, sendo 8 alunos do sexo masculino e 9 do sexo feminino (GRAFICO $1)$.

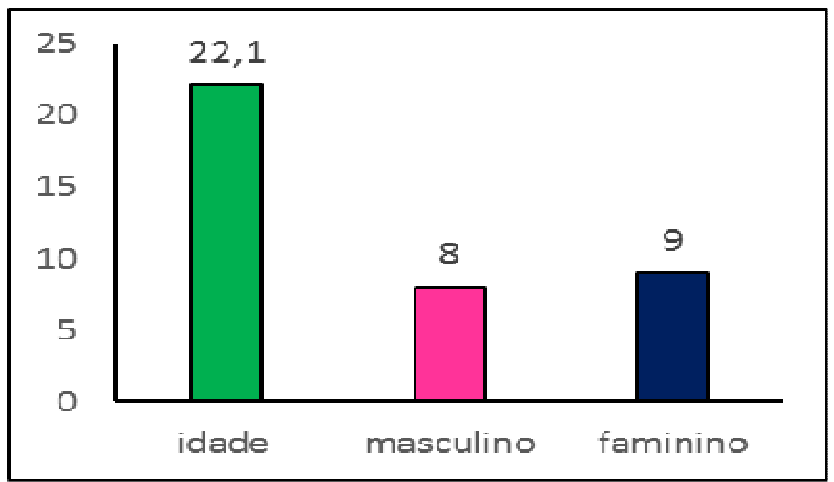

Gráfico 01- Variáveis referentes a média de idade e caraterização de gênero nos discente

Fonte: Pesquisa de Campo, 2014. 
A seguir apresenta- se os dados referentes a analise ergonômica dos discentes por meio mapa de desconforto corporal RULA, OWAS.

No mapa de desconforto corporal foram analisadas as regiões onde os acadêmicos sentem dor durante os atendimento fisioterapêuticos, sendo que um acadêmico podia sentir dor em uma ou mais regiões do corpo e foram encontrados os seguintes resultados, $11(64,7 \%)$ acadêmicos sentem dor em coluna lombar, 6 (35,9\%) acadêmicos sentiam dor em coluna torácica, 5 $(29,4 \%)$ acadêmicos sentem dor em coluna cervical, $1(5,8 \%)$ acadêmico sentia dor em sacro, $1(5,8) \%$ sentia dor em punho, ilustrado no gráfico 2.

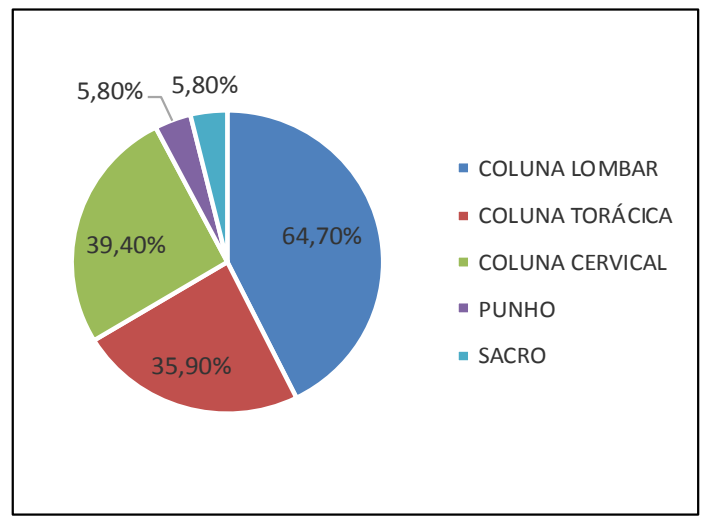

Gráfico 02 -Mapa de Desconforto Corporal Fonte: Pesquisa de Campo, 2014

$\mathrm{Na}$ análise postural foram selecionadas 41 posturas mais frequentes durante os atendimento, então foram aplicadas as ferramentas RULA e o OWAS quantificado seus graus de riscos de acordo com os scores obtidos ao final da análise.

Em relação ao RULA a média do score final das posturas foram 4,2 onde
Dentre os quatro níveis de ação encontraramse os seguintes resultados: 6 posturas $(14,6 \%)$ encontravam-se no nível 1 (pontuação 1-2) aceitável, 15 postura $(36,6 \%)$ encontravam se no nível 2 (pontuação 3-4) requer mudanças, $18(44 \%)$ posturas encontravam-se no nível 3 (pontuação 5-6) requer mudanças rapidamente, 2 posturas $(4,8 \%)$ encontravamse no nível 4 (pontuação 7+) requer mudanças imediatamente.

Tabela 01- Dados referentes ao score final do método RULA

\begin{tabular}{|l|l|l|l}
\hline $\begin{array}{l}\text { Score } \\
\text { final }\end{array}$ & $\begin{array}{l}\mathbf{N}^{\mathbf{0}} \mathbf{d e} \\
\text { posturas }\end{array}$ & Resultado Média & DP \\
\hline $\begin{array}{l}\text { Nível 1 } \\
(\mathbf{1 - 2})\end{array}$ & 6 & Aceitável \\
\hline $\begin{array}{l}\text { Nível 2 } \\
(\mathbf{3}-4)\end{array}$ & 15 & $\begin{array}{l}\text { Requer } \\
\text { mudanças }\end{array}$ \\
\hline $\begin{array}{l}\text { Nível 3 } \\
(\mathbf{5 - 6 )}\end{array}$ & 18 & $\begin{array}{l}\text { Requer } \\
\text { mudanças } \\
\text { rapidamente }\end{array}$ \\
\hline $\begin{array}{l}\text { Nível 4 } \\
\text { (7) }\end{array}$ & 2 & $\begin{array}{l}\text { Requer } \\
\text { mudanças } \\
\text { imediatamen } \\
\text { te }\end{array}$ \\
\hline
\end{tabular}

Fonte: Pesquisa de Campo, 2014

No método OWAS a média do score final das posturas foi 1,7 sendo 15 posturas (36,5\%) obtendo o score final 1 (não são necessários medidas corretivas), 24 posturas $(58,5 \%)$ obtendo score final 2 (são necessárias medidas corretivas), 1 postura $(2,5 \%)$ obtendo o score final 3 (são necessárias correções tão logo quando possível) e 1 postura (2,5\%) obtendo o score final 4 (são necessárias correções imediatas). 
Tabela 02- Dados referentes ao score final do método OWAS

\begin{tabular}{|c|c|c|c|c|}
\hline $\begin{array}{l}\text { Score } \\
\text { final }\end{array}$ & $\begin{array}{l}\mathbf{N}^{\mathbf{0}} \text { de } \\
\text { posturas }\end{array}$ & Resultado & Média & DP \\
\hline 1 & 15 & $\begin{array}{l}\text { Não são } \\
\text { necessários } \\
\text { medidas } \\
\text { corretivas }\end{array}$ & \multirow{4}{*}{1,7} & \multirow{4}{*}{0,6} \\
\hline 2 & 24 & $\begin{array}{l}\text { São } \\
\text { necessárias } \\
\text { medidas } \\
\text { corretivas }\end{array}$ & & \\
\hline 3 & 1 & $\begin{array}{l}\text { São } \\
\text { necessárias } \\
\text { correções tão } \\
\text { logo quando } \\
\text { possível }\end{array}$ & & \\
\hline 4 & 1 & $\begin{array}{l}\text { São } \\
\text { necessárias } \\
\text { correções } \\
\text { imediatas }\end{array}$ & & \\
\hline
\end{tabular}

Fonte: Pesquisa de Campo, 2014

\section{DISCUSSÃO}

Os resultados obtidos nesta pesquisa revelaram uma prevalência de distúrbios posturais nos acadêmicos de fisioterapia. Apesar de haver poucas literaturas que abordem o assunto há um consenso que acadêmicos de fisioterapia sofrem processos álgicos durante o atendimento. Os dados obtidos pela aplicação do Método RULA na avaliação postural, confirmou estes resultados demonstrando a necessidade das modificações a serem tomadas para esses futuros profissionais da saúde.

A Norma Regulamentadora NR-17, estabelece parâmetros que permitem a adaptação das condições de trabalho às características psicofisiológicas dos trabalhadores, de modo a proporcionar um máximo de conforto, segurança e desempenho eficiente.
Muitas vezes o trabalho com seres humanos não permite a aplicação do conceito do princípio ergonômico em que necessita de “adequação do trabalho ao trabalhador", pois cada paciente é visto de forma individual e com diferenças antropométricas que variam em cada paciente, segundo Hignett (1995) houve a difusão da crença em que o paciente sempre vem em primeiro lugar, podendo então ocasionar negligência na adoção de posturas e técnicas a serem realizadas. Sendo assim, é importante reconhecer a vulnerabilidade da profissão e assim então adequar e selecionar as técnicas mais adequadas durante o trabalho.

A forma mais fácil para a realização da avaliação ergonômica das tarefas, ou postos de trabalho, no que se refere aos riscos posturais é através de métodos expeditos desenvolvidos para este fim, dentre esses métodos encontra-se o OWAS método que apresenta os resultados das porcentagens de tempo que o trabalhador permanece em uma postura "boa" e "má", e ainda propiciar o direcionamento para a melhoria do posto de trabalho e o RULA método desenvolvido para investigar a exposição dos trabalhadores aos fatores de risco associados aos distúrbios dos membros superiores (CARDOSO, 2006).

Em relação aos tipos de postura adotada no momento do atendimento obtevese que cerca de $70 \%$ das posturas encontradas apresentavam flexão e rotação de tronco e 
cervical, isso pode explicar o aparecimento de dores cervical e lombar, e Carregaro et al (2006) diz que a fisioterapia pode ser uma ocupação estressante principalmente quando relacionados aos fatores como dor lombar, membros inferiores, mão, punhos e polegar, sendo um ocupação que utiliza de esforço físico e envolve algumas atividades como levantamento, inclinação, flexão e rotação de tronco, manutenção da posição de posturas inadequadas, podendo ser fatores que originam distúrbios musculares relacionados ao trabalho.

Os diversos recursos utilizados dentro da fisioterapia, dentre eles os recursos manuais, requerem dos acadêmicos e profissionais diversas posições e variadas aplicações de forças, aumentando ainda os riscos de incidência de sobrecargas posturais, segundo Peres (2002), dentre as atividades exercidas pelo fisioterapeuta, destacam-se as terapias manuais com inclinação de tronco para frente, rotação de tronco, membros superiores em variadas posições, ora acima ou ora abaixo dos níveis do ombro, muitas vezes com sustentação e carga, acarretando alteração na biomecânica corporal, desencadeando fadigas musculares e provavelmente lesões ostemioarticulares, concordando com o resultado das regiões de dores do acadêmicos pelo mapa de desconforto corporal e os achados utilizando o método RULA.
Carregaro et al (2006) em seu estudo cujo o objetivo foi verificar a frequência, fatores de risco, áreas de atuação e decorrências de lesões ocupacionais entre fisioterapeutas, trouxe que dentre os estudos que relacionaram as áreas de atuação com os sintomas, foi encontrada elevada prevalência de LER/DORT em fisioterapeutas atuantes da área de neurologia, ortopedia, reumatologia, cardio-pneumo, hidroterapia e geriatria, áreas de concentração do estágio ambulatorial de fisioterapia dos acadêmicos.

Dantas (2006) em sua pesquisa com 20 acadêmicos de fisioterapia onde um dos objetivos do trabalho foi analisar quantitativamente dores que acometem acadêmicos de fisioterapia após atendimentos de pacientes com disfunções neurológicas onde verificou que $63 \%$ relataram dor em região lombar, $25 \%$ na região lombar e cervical concomitantemente e $6 \%$ na região cervical e membros superiores (MMSS) e 6\% na lombar e MMSS.

Guedes (2008) em sua pesquisa com 45 acadêmicos de fisioterapia, onde um dos objetivos foi demonstrar qual área da coluna vertebral é mais acometida pela dor verificou que as regiões mais afetadas da coluna foram lombar correspondente a 64, 28\%, e em região cervical $23,21 \%$, em região torácica $10,71 \%$, equiparados aos resultados obtidos nesse estudo. 
Neste estudo, resultados encontrados mapa de desconforto corporal evidenciou que os acadêmicos de fisioterapia durante os atendimentos, apresentaram dores em diferentes partes do corpo sendo mais presente em regiões de coluna vertebral sendo que um acadêmico pode sentir dor em mais de uma região do corpo, as regiões mais afetadas foram coluna lombar, coluna torácica, coluna cervical, sacro e punho, corroborando com os achados de Dantas (2006) e Guedes (2008).

No método OWAS que avalia principalmente membros inferiores a média do score final das posturas foi 1,7 sendo que a maior prevalência foi o score final 2. De acordo com o método OWAS as posturas não apresentaram um nível alto de risco, porém são necessárias algumas mudanças. Peres (2002) em sua pesquisa onde o objetivo foi analisar a incidência das sobrecargas posturais em fisioterapeutas, decorrentes das posturas adotadas nos procedimentos terapêuticos durante as atividades de trabalho, constatou utilizando o método OWAS que as posturas adotadas durante os procedimentos analisados enquadram-se: $39 \%$ na categoria 2, $26 \%$ na categoria $1,18 \%$ na categoria 3 e $16 \%$ na categoria 4, mostrando a importância de algumas mudanças nas posturas adotadas por fisioterapeutas no trabalho, sendo que as atividades dos acadêmicos do último período do curso de fisioterapia são voltadas para a prática profissional se equiparando ao trabalho desses profissionais.
Na análise pelo método RULA obtevese a média 4,2 dos scores das posturas adotadas, sendo que as analises das posturas evidenciou um alto índice de sobrecargas posturais onde $44 \%$ das posturas analisadas encontra-se no nível 3 onde se estimam mudanças rapidamente e $36,6 \%$ no nível 2 onde se requer mudanças, esses resultados demonstram evidências no surgimento de distúrbios posturais nos acadêmicos. Até o momento não se elucidou em artigos científicos analises semelhantes corroborando ou não com os dados deste estudo.

\section{CONCLUSÃO}

Os resultados obtidos neste estudo observa-se uma prevalência de dores principalmente na coluna vertebral e distúrbios posturais nos acadêmicos de Fisioterapia durante suas atividades de trabalho no estágio supervisionado na Clínica Escola de Fisioterapia do CESUPA. Mesmo sendo preparado durante toda sua vida acadêmica para cuidar dos distúrbios apresentados pelos pacientes, eles podem não estar preparado para utilizar determinadas posturas nas suas atividades profissionais diárias, podendo ocasionar desgaste físico e descompensatório.

Foi notório que os acadêmicos sofrem uma sobrecarga postural durante o atendimento, acarretando em dores, muitas vezes se preocupando com o bem estar do 
paciente e esquecendo-se do seu. A compreensão da biomecânica corporal durante os atendimentos é importante para que os acadêmicos assim possam adotar posturas mais adequadas como mecanismo de defesa do sistema osteomioarticular para cada situação, atendendo seu paciente de forma integral sem gerar nenhuma forma de agressão a si mesmo.

A realização de técnicas manuais com esforço, associados à flexão e rotação de tronco e esforços dos membros superiores é frequentemente utilizada por esses profissionais. É importante ressaltar a conscientização desde a formação acadêmica desses futuros profissionais; enfatizar as possíveis alterações corporais que podem ser causadas por uso repetido de posturas inadequadas utilizadas durante o atendimento, podendo causar um grande prejuízo na sua vida profissional, sugerindo-se a adoção medidas preventivas e orientações quanto a posturas e condições de trabalho.

\section{REFERENCIAS}

CARDOSO, M. M. J.; Avaliação

Ergonômica: Revisão dos Métodos para Avaliação Postural, R. Eletr. de Eng. de Produção e Correlatas, Florianópolis,V 6, N 3 ,2006 Disponível em: http://producaoonline.org.br/rpo/article/view/ 630/668. Acesso em: 03 de Agosto de 2013.
CARREGARO, R.S.; TRELHA, C.S.;

MASTELARI, H.J. Distúrbios

osteomusculares relacionados ao trabalho em fisioterapeutas: revisão da literatura, Fisioterapia E Pesquisa,V13, N 1, P: 53-59, 2006

DANTAS, M.D.F.; NASCIMENTO, A.C.L.; TORRES, C.K.D.; MOREIRA, K.L.A.F.; COSTA, S.M.L.

Análise Da Dor Nos Acadêmicos De Fisiotera pia Da Universidade Federal

Da Paraíba Após Atendimento A Pacientes C om Disfunções Neurológicas, 2006

\section{DUARTE, M; SANDRA, MSF. Revisão} sobre posturografia baseada em plataforma de força para avaliação do equilíbrio, Revista Brasileira Fisioterapia, São Carlos, V14, 3, P:183-192, 2010. Disponível em : http://www.scielo.br/pdf/rbfis/v14n3/03.pdf Acesso em: 30 de agosto de 2013

FONTES, C.F.; RAVAGNANI, I.L.M.; ZAIA J.E.; QUEMELO, P.R.V. Comparação da sobrecarga mecânica em funcionários que executam suas tarefas na posição em pé e sentada, Revista brasileira Ciencia e Movimento, V 21,N1, P:10-15, 2013.

Disponível em: portalrevistas.ucb.br/index.php/RBCM/article /viewFile/3294/2465. Acesso em:30 de agostode 2013

GUEDES, F.G.; MACHADO, A.P.N.B.

Fatores que influenciam no aparecimento das 
dores na coluna vertebral de acadêmicos de fisioterapia. Estação Científica Online. Juiz de Fora, n. 05, Jan. 2008. Disponível em

:<http://portal.estacio.br/media/3304416/4fatores-que-influenciam-aparecimento-dorescoluna-vertebral-academicos-

fisioterapia.pdf >. Acesso em: 03 de Agosto de 2013.

HIGNETT S. Fitting the work to the physiotherapist. Physiotherapy, V81, N 9, P:549-52, 1995

KALSING K. Fatores de risco para desenvolver dor musculoesquelética. Trabalho de Conclusão de Curso (Graduação em Enfermagem)- Universidade Federal do Rio Grande do Sul, Porto Alegre, 2010. Disponível em: http://www.lume.ufrgs.br/handle/10183/2471 0. Acesso em: 01 de Setembro de 2013.

MACHADO, N.P.; RATTS, R.C.M.R.; NOGUEIRA, V.C.; COSTA, A.S.M.;

MARTINS, M.; ANSAWA, E.A.L. Incidência de LER/ DORT em fisioterapeutas docentes de uma instituição de ensino superior em Teresina (PI).IN: XIII Encontro Latino Americano de Iniciação Científica e IX Encontro Latino Americano de PósGraduação - Universidade do Vale do Paraíba, 2009. Disponível em: http://www.inicepg.univap.br/cd/INIC_2009/a nais/arquivos/RE_0350_0345_01.pdf. Acesso em: 01 de Setembro de 2013.
MAEHLER P. Estudo das sobrecargas posturais em Acadêmicos de odontologia da universidade Estadual do Oeste do Paraná Unioeste - Cascavel ,monografia ( trabalho de graduação),cascavel, 2003. Disponível em:http://www.unioeste.br/projetos/elrf/mono grafias/2003/mono/14.pdf . Acesso em: 10 de agosto de 2013

\section{MAGALHÃES, O.S.V.M.; MONTEIRO,} R.S.; RODRIGUES, C.C.W. Análise Da Aplicabilidade Da Fisioterapia Preventiva, Através Dos Princípios E Exigências Ergonômicas, À Odontologia: Revisão Da Literatura, Rev. Científica ESAMAZ, Belém, V 3, N 1, 2011

MARQUES, N.R.; HALLAL, C.Z.;

GONÇALVES, M. Características biomecânicas, ergonômicas e clínicas da postura sentada: uma revisão, Revista Fisioterapia e movimento, São Paulo, V 17, N 3, P: 270-276, 2010. Dísponível em: http://www.scielo.br/pdf/fp/v17n3/15.pdf Acesso em: 28 de agosto de 2013

MORAES G. Legislação de Segurança e Saúde no Trabalho,10 ED., VOL 1,EDITORA GVC, 2013

[MTE] Ministério do Trabalho e Emprego. Norma Regulamentadora NR-17 Ergonomia. Disponível em: http://www.mte.gov.br/Empregador/segsau/L egislacao/Normas/conteudo/nr17/ 
PERES, CPA Estudo Das Sobrecargas

Posturais Em Fisioterapeutas: Uma

Abordagem Biomecânica Ocupacional,

Florianópolis, 2002, f.128 , dissertação (

Mestre em engenharia de produção),

programa de mestrado em engenharia de

produção, Universidade Federal de Santa

Catarina, 2002. Disponível

em:http://escudine.com.br/txt/ART/estudo\%2

0das $\% 20$ sobrecargas $\% 20 \mathrm{de} \% 20$ postura $\% 20 \mathrm{p}$

ara\%20fisioterapeutas.pdf. Acesso em: 26 de

Agosto de 2013.

ZAPATER, A.R. et al. Postura sentada: a

eficácia de um programa de educação para

escolares , Ciência e saúde coletiva, Rio de

Janeiro, V 9, N 1, P: 191-199, 2004. 